\title{
Special issue with contributions to the Fifth Baltic Conference on Electrochemistry: functional materials in electrochemistry-from fundamental problems to molecular electronics and modern power sources (30 April-3 May 2008, Tartu, Estonia)
}

\author{
Enn Lust \\ Received: 18 November 2008 / Accepted: 18 November 2008 /Published online: 11 December 2008 \\ (C) Springer-Verlag 2008
}

The conference under discussion was the fifth event in the series of international Baltic electrochemistry conferences started in 1997 (Tartu, Estonia). The conference was realised in traditional form including one plenary (A.A. Kornyshev) and nine invited lectures from world-leading specialists (J. Ulstrup, Th. Wandlowski, R.R. Nazmutdinov, Y. Gogotsi, S. Singhal, E.A. Kotomin, A. Wieckowski, D. Scherson, U. Stimminghowever given by A.A. Kornyshev) discussing various fundamental and applied electrochemistry problems including quantum chemical theories of charge transfer, 2D-layer structure and adsorption layer formation kinetics, carbonbased supercapacitors, polymer electrolytes, solid oxide fuel cells and modern surface analysis methods in electrochemistry. In addition, there were 37 very interesting oral lectures and also 53 poster presentations. So, the conference covered various aspects of materials science and surface physics connected with the problems of modern electrochemistry.

The main topics of the discussions during the conference were the role of electrochemistry for variable optics, future development of the supercapacitors with higher energy density by using the room temperature ionic liquids, new solvent systems; possibilities to decrease the price of proton exchange membrane fuel cells through the catalyst and membrane design. Also, time-durability problems of new materials for solid oxide and microbial fuel cells have been analysed. The importance of the complex analysis, including quantum chemical simulations of the surface chemistry and different modern physical methods (in situ neutron diffraction, X-ray photoelectron spectroscopy, secondary ion mass spectrometry, infrared synchrotron radiation), has been notified. The surface dynamics of the well-defined electrodeelectrolyte interface, the reactivity of Pt-metal nanoclusters and particle size effects have been discussed very enthusiastically by the participants of the conference.

The actual special issue represents the original publications from participants of the Fifth Baltic Conference on Electrochemistry.

E. Lust $(\bowtie)$

Institute of Physical Chemistry, University of Tartu,

Jakobi 2 ,

51014 Tartu, Estonia

e-mail: enn.lust@ut.ee 